\title{
Deformation effects in low-momentum distributions of heavy nuclei
}

\author{
V.O. Nesterenko ${ }^{1,2}$, V.P. Likhachev², P.-G. Reinhard ${ }^{3}$, \\ J. Mesa ${ }^{2}$, W. Kleinig ${ }^{1,4}$, J.D.T. Arruda-Neto ${ }^{2,5}$, and A. Deppman ${ }^{2}$ \\ 1 Bogoliubov Laboratory of Theoretical Physics, \\ Joint Institute for Nuclear Research 141980, Dubna, \\ MoscowRegion, Russia, E-mail: nester@thsun1.jinr.ru \\ 2 Instituto de Física, Universidade de São Paulo, São Paulo, Brazil \\ 4 Institut für Theoretische Physik, Universität Erlangen, D-91058, Erlangen, Germany \\ 4 Technische Universität Dresden, Institut für Analysis, Dresden, D-01062, Germany and \\ 5 Universidade de Santo Amaro, São Paulo, Brazil
}

\begin{abstract}
Momentum distributions (MD) of deep hole proton states in ${ }^{238} \mathrm{U}$ are studied paying particular attention to the influence of deformation. Two essentially different mean-field models, WoodsSaxon (WS) and Skyrme-Hartree-Fock with the SkM* force, are used. Noticeable deviations between the WS and $\mathrm{SkM}^{*}$ results are found. They are mainly due to the difference in effective nucleon mass. In particular, $\mathrm{SkM}^{*}$ gives much weaker deformation effects at low momenta than WS. It is shown that, in spite of the deformation mixing, MD at low momenta can serve for identification of $K^{\pi}=1 / 2^{+}$hole states originating from $s_{1 / 2}$ spherical sub-shells. Moreover, following the WS calculations, the deformation results in additional $K^{\pi}=1 / 2^{+}$states with strong $l=0$ contributions. A possibility to probe such states in knock-out experiments is discussed.
\end{abstract}


The momentum distribution (MD) of nucleons in a nucleus is an important issue for analyzing single-particle aspects of nuclear structure, for a review see [1]. One of the still open problems is the influence of global nuclear deformation on MD. There exists already some studies on that subject, see, e.g. [2] and references. therein. Nonetheless, the present knowledge about deformation effects in MD is still rather poor, especially in heavy deformed nuclei. At the same time, these effects can be essential for a correct treatment of knock-out reactions, like $\left(e, e^{\prime} p\right)$, in rare earth and actinide regions and for investigation of deep hole states in deformed nuclei (see, e.g. [3]).

Experimental data on deep hole states are scarce in spherical nuclei and practically absent in heavy deformed nuclei [1]. The deformation mixes spherical configurations in singleparticle wave functions [4] and thus should influence the MD. At the first glance, this effect, together with a dense spectrum in deformed nuclei and possible coupling with vibrations, should make MD too vague to extract from them a reliable information about single-particle levels. However, as is shown below, there remains a chance to identify some hole $K^{\pi}=1 / 2^{+}$ levels through their MD at low momenta. Moreover, the deformation mixing can increase the number of such levels. Experimental observation of the $K^{\pi}=1 / 2^{+}$level sequence in $\left(e, e^{\prime} p\right)$ could be a very important step in our knowledge on the mean field in heavy deformed nuclei. Besides, it could have a serious impact in such urgent problems, as, e.g., a search for shell-stabilized super-heavy nuclei [5].

The present paper pursues two aims. First, we will try to exhibit, at least briefly, a general view of deformation effects in deep hole MD of heavy deformed nuclei. To make the study more reliable, two different single-particle potentials, phenomenological Woods Saxon and self-consistent Skyrme, will be used. Second, we will demonstrate that low momentum distributions in heavy deformed nuclei represent a relevant test for different models and deserve a careful experimental investigation.

The calculations have been performed for ${ }^{238} \mathrm{U}$, a typical heavy deformed nucleus. Two different models for single-particle structure were used: the phenomenological Woods-Saxon (WS) potential [6, 7] with parameters from [8] and the self-consistent Skyrme-Hartree-Fock potential 9] with the parametrization $\mathrm{SkM}^{*}$ [10]. We have chosen $\mathrm{SkM}^{*}$ as a widely established standard well suited to describe deformed nuclei $\left(\mathrm{SkM}^{*}\right.$ was fitted to describe fission barriers in actinide region). The WS calculations were performed in a basis expansion while the $\mathrm{SkM}^{*}$ calculations employed an axially symmetric coordinate-space grid. For both WS 
TABLE I: Quadrupole moments $\left(Q_{2}\right)$, Fermi energy $\left(E_{F}\right)$ and the lowest $\left(E_{0}\right)$ single-particle levels in ${ }^{238} U$. Experimental estimations for the quadrupole moment in ${ }^{238} \mathrm{U}$ lie in the interval $Q_{2}=11.1-11.3 \mathrm{~b}[11,12]$.

\begin{tabular}{|c|c|c|c|}
\hline Potential & $Q_{2}[\mathrm{~b}]$ & $E_{F}[\mathrm{MeV}]$ & $E_{0}[\mathrm{MeV}]$ \\
\hline WS & 11.0 & -7.5 & -33.7 \\
SkM $^{*}$ & 11.1 & -6.2 & -39.8 \\
\hline
\end{tabular}

and $\mathrm{SkM}^{*}$, the equilibrium ground-state deformations were determined by minimizing the energy of the system. MD were defined as single-particle densities $n_{\alpha}(\mathbf{k})=|\tilde{\varphi}(\mathbf{k})|^{2}$ in momentum space. They were obtained by Fourier transforming the spatial wavefunctions, for procedure and definitions see [3]. It is worth noting that in general single-particle models are not well suited to describe MD because of the important contributions from short- and long-range correlations [1]. However these perturbing effects take place mainly in the highmomentum domain with $k>k_{\mathrm{F}} \approx 1.3 \mathrm{fm}^{-1} \approx 260 \mathrm{MeV} / \mathrm{c}$. We will focus on MD in the low $k$ domain, which can be still described by single-particle models.

As is seen from table I both single-particle models reproduce nicely the ground state deformation (quadrupole moments) in ${ }^{238} \mathrm{U}$. The Fermi energy $E_{F}$ is also nearly the same in both models. However, the lowest proton hole state $E_{0}$ is much deeper bound for SkM*. The difference in $E_{0}-E_{F}$ indicates a different level density. This is also visible in the single proton levels shown in table【. The SkM* spectrum is less dense because of the lower effective mass $m^{*} / m=0.79$ as compared to $m^{*} / m=1$ in the WS model. Note that table II] shows only a subset of the levels whose MD will be discussed further in more detail.

Figure 1 shows the calculated MD for six deep hole proton states given in the table III. These states represent a selection with different energies and quantum numbers. SkM* results are given for the equilibrium deformed ground state and, for reasons of comparison, in the spherical limit produced by constraint. One sees that in both these cases MD are very close. Modest changes are caused by the deformation at low momenta in $1 / 2^{-}[321]$, $1 / 2^{+}[440]$, and $1 / 2^{-}$[541], i.e. in states with a minimal projection $K$. At the same time, as illustrated by the plots for $5 / 2^{-}[312]$ and $3 / 2^{+}[422]$, MD of the hole states with $K \geq 3 / 2$ are almost unaffected. The latter can be easily explained by the fact that states with high 
TABLE II: Energies of selected proton hole states in ${ }^{238} \mathrm{U}$, calculated with WS and $\mathrm{SkM}^{*}$ potentials. The lower part of the table collects all the hole $K_{i}^{\pi}=1 / 2^{+}$states. For every state the spectroscopic quantum numbers of the spherical ancestor are given. The low index in $K_{i}^{\pi}$ numerates states with a given $K^{\pi}$ from the bottom of the wall.

\begin{tabular}{|c|c|c|c|}
\hline \multirow{2}{*}{$K_{i}^{\pi}\left[N n_{z} \Lambda\right]$} & \multirow{2}{*}{$n l j$} & \multicolumn{2}{|c|}{$\mathrm{E}, \mathrm{MeV}$} \\
\cline { 3 - 4 } & & $\mathrm{WS}$ & $\mathrm{SkM}^{*}$ \\
\hline $1 / 2_{8}^{-}[541]$ & $1 h_{11 / 2}$ & -10.4 & -9.5 \\
$3 / 2_{4}^{+}[422]$ & $1 g_{7 / 2}$ & -14.3 & -14.7 \\
$1 / 2_{6}^{-}[301]$ & $2 p_{1 / 2}$ & -15.5 & -16.4 \\
$5 / 2_{1}^{-}[312]$ & $1 f_{7 / 2}$ & -21.3 & -23.3 \\
$1 / 2_{4}^{-}[321]$ & $1 f_{5 / 2}$ & -21.6 & -23.9 \\
\hline \hline $1 / 2_{10}^{+}[660]$ & $1 i_{13 / 2}$ & -8.3 & -6.8 \\
$1 / 2_{9}^{+}[400]$ & $3 s_{1 / 2}$ & -7.2 & -6.9 \\
$1 / 2_{8}^{+}[411]$ & $2 d_{3 / 2}$ & -9.8 & -9.7 \\
$1 / 2_{7}^{+}[420]$ & $2 d_{5 / 2}$ & -13.1 & -13.5 \\
$1 / 2_{6}^{+}[431]$ & $1 g_{7 / 2}$ & -16.4 & -17.1 \\
$1 / 2_{5}^{+}[440]$ & $1 g_{9 / 2}$ & -19.3 & -20.5 \\
$1 / 2_{4}^{+}[200]$ & $2 s_{1 / 2}$ & -22.8 & -25.4 \\
$1 / 2_{3}^{+}[211]$ & $1 d_{3 / 2}$ & -26.0 & -29.5 \\
$1 / 2_{2}^{+}[220]$ & $1 d_{5 / 2}$ & -28.1 & -32.1 \\
$1 / 2_{1}^{+}[000]$ & $1 s_{1 / 2}$ & -33.7 & -39.8 \\
\hline
\end{tabular}

$K$ have lower level density and so less chances for deformation mixing.

Figure 1 compares also SkM* and WS results. Unlike SkM*, WS gives impressive deformation effects at low momenta for the $1 / 2^{-}[321], 1 / 2^{+}[440], 3 / 2^{+}[422]$, and $1 / 2^{-}[541]$ states, where one-bump spherical MD are transformed by deformation to more complex structures. The discrepancies between WS and $\mathrm{SkM}^{*}$ can be explained, to a large extent, by the lower effective mass $m^{*} / m=0.79$ in $\mathrm{SkM}^{*}$. This stretches the single-particle spectrum [14] for $\mathrm{SkM}^{*}$ and thus weakens the deformation effects.

As was mentioned above, single-particle models are not reliable in the high-momentum 
domain with $k>260 \mathrm{MeV} / \mathrm{c}$. Nevertheless, it is worth to comment on some basic trends of the models as such, hinted by high-momentum MD. First, as compared with SkM*, the MD of WS are characterized by a stronger high-momentum tail and thus by a smaller peak height at $k \sim 170 \mathrm{MeV} / \mathrm{c}$. Second, $\mathrm{SkM}^{*}$ results for $1 / 2^{+}[440]$ and $1 / 2^{-}[541]$ show that the deformation can influence the high-energy MD tail.

Figure 1 shows that MD for $1 / 2^{+}[440]$ is peaked at $k=0$ thus manifesting an appreciable $l=0$ component. This feature can essentially facilitate identification of the hole $K^{\pi}=1 / 2^{+}$ levels in knock-out reactions. We are thus inspecting these states in more detail. Figure 1 collects MD for all hole $K^{\pi}=1 / 2^{+}$levels in ${ }^{238} \mathrm{U}$ (the spectroscopic information is given in table II). It is seen that WS calculations predict an appreciable $l=0$ strength (peak at $k=0$ ) for 7 states, namely for all those with $\Lambda=0$. The maximal strength takes place for states $1 / 2^{+}[000], 1 / 2^{+}[200]$, and $1 / 2^{+}[400]$ originating from $1 s, 2 s$, and $3 s$ spherical subshells, respectively. The other four states, $1 / 2^{+}[220], 1 / 2^{+}[440], 1 / 2^{+}[420]$, and $1 / 2^{+}[660]$, have higher $l$ ancestors but acquire such a feature due to the admixture of $l=0$ spherical subshells, caused by the deformation mixing. So, the deformation leads to an increased number of $K^{\pi}=1 / 2^{+}$states with a strong $l=0$ component. As was mentioned above, SkM* produces much weaker deformation effects. Indeed, $\mathrm{SkM}^{*}$ gives $k=0$ peaks only for the states originating from $1 s, 2 s$, and $3 s$ spherical subshells, being consistent in this case with the WS results. The $l=0$ strength for other states is negligible.

Altogether, figure 2 indicates that MD at low momenta allow to extract important information on the underlying mean field. Namely, a sequence of hole $K^{\pi}=1 / 2^{+}$levels can be found experimentally. The question is whether one can resolve them. In the following, we will analyze this point in more detail.

Figure 3 shows the calculated energy distribution of MD strength at $k=0$ for the hole $K^{\pi}=1 / 2^{+}$levels. It is seen that $\mathrm{SkM}^{*}$ and WS represent two different scenarios. In the $\mathrm{SkM}^{*}$ case, only $1 / 2^{+}[000], 1 / 2^{+}[200]$, and $1 / 2^{+}[400]$ levels originating from $1 s$, $2 s$, and $3 s$ spherical subshells and giving the biggest $k=0$ peaks can be resolved. The picture looks like in heavy spherical nuclei. Indeed, in the ${ }^{208} \mathrm{~Pb}\left(e, e^{\prime} p\right)$ reaction, the $l=0$ strength was observed only as the ground state $3 s_{1 / 2}$ and deep hole $2 s_{1 / 2}$ structures [13]. The former was found as a strong narrow peak and the latter as a broad bump spreaded in the interval 7-8 MeV with the centroid at $\sim 21.3 \mathrm{MeV}$ (the $1 s_{1 / 2}$ was beyond the energy interval covered in the experiment). WS calculations predict more interesting situation when at least 
three additional levels $\left(1 / 2^{+}[440], 1 / 2^{+}[420]\right.$, and $\left.1 / 2^{+}[660]\right)$ placed between $1 / 2^{+}[200]$ and $1 / 2^{+}[400]$ have a chance to be observed (more deeply bound levels can hardly be indicated because of a too strong broadening expected for them).

Single particle energies in mean field models have well defined discrete values as indicated by the simple bars in figure 3. However, l-strengths measured in $\left(e, e^{\prime} p\right)$ experiments are spreaded, becoming broader while going away from the Fermi energy towards deeply bound states. The spread is caused by correlations beyond the mean field approximation, in particular by the coupling the holes with 1ph configurations and vibrations (see [1, 13, 14] and references therein). The deformation also contributes to the spread. Knock-out reactions are sensitive only to single-hole components which are the door-way states for them. So, the MD signal at $k=0$ will not be masked by complex components of the wave function, caused by the correlations. But the signal will be smeared out energetically to a more or less broad bump corresponding to a certain $K^{\pi}=1 / 2^{+}$level. The question is whether these bumps overlap each other or they are still separated enough to allow identification of the various $K^{\pi}=1 / 2^{+}$levels in $\left(e, e^{\prime} p\right)$ reaction.

As is seen from figure 3, in the $\mathrm{SkM}^{*}$ calculations the relevant levels $1 / 2^{+}[200]$ and $1 / 2^{+}[400]$ are well separated in energy and so, in spite of a (weak) deformation mixing, can be observed. In the WS calculations, the levels $1 / 2^{+}[200], 1 / 2^{+}[440], 1 / 2^{+}[420], 1 / 2^{+}[660]$, and $1 / 2^{+}[400]$ are separated by the intervals $3-6 \mathrm{MeV}$ (which hint at the energy scale for the deformation mixing). Following the ${ }^{208} \mathrm{~Pb}\left(e, e^{\prime} p\right)$ data [13], the correlation spread should not exceed 7-8 MeV and for most of the states (as less bound) should even much weaker. For these states the correlation and deformation spreads seem to be of the same order of magnitude. So, we probably have an interesting situation when the deformation does not noticeably hinder the identification of $l=0$ structures but instead can increase the number of such structures.

In summary, momentum distributions (MD) for single-hole proton states in ${ }^{238} \mathrm{U}$ were analyzed within two different mean field models: phenomenological Woods-Saxon (WS) and self-consistent Skyrme-Hartree-Fock with the force SkM*. WS gives much stronger deformation effects than $\mathrm{SkM}^{*}$, which is explained by the lower effective mass in SkM* (in fact $\mathrm{SkM}^{*}$ description stays close to the spherical case). The deformation mainly influences $K^{\pi}=1 / 2^{+}, 1 / 2^{-}$hole states in the low momentum domain. WS results predict a sequence of $K^{\pi}=1 / 2^{+}$states with strong $l=0$ contributions. Some of these states acquire the 
$l=0$ strength due to the deformation mixing. The property of $l=0$ strength to give a peak in MD at zero momenta considerably simplifies identification of $l=0$ structures. Our analysis shows that the $K^{\pi}=1 / 2^{+}$states have a good chance to be discriminated in knock-out reactions. Observation of a sequence of such states in heavy deformed nuclei would provide a valuable information about their mean field. Besides, experimental study of the low-momentum MD in heavy deformed nuclei would help to discriminate the various mean-field models.

Acknowledgment: The work was partly supported by grants from FAPESP (Grant No. 2001/06082-1), RFBR (Grant No. 00-02-17194), and Germany-BLTP JINR ( HeisenbergLandau). We express also our gratitude to Profs. A.N. Antonov, A.V. Afanasjev and J.R. Marinelli for their useful comments.

[1] A.N. Antonov, P.E. Hodgson, and I.Zh. Petkov, Nucleon Correlations in Nuclei, SpringerVerlag Berlin Heidelberg, 1993.

[2] E. Moya de Guerra, J.A. Caballero, and P. Sarriguren, Nucl. Phys. A477, 445 (1988).

[3] V.P. Likhachev, J. Mesa, J.D.T. Arruda-Neto, B.V. Carlson, A. Deppman, M.S. Hussein, V.O. Nesterenko, F. Garcia, and O. Rodriguez, Phys. Rev. C65, 044611 (2002).

[4] F.A. Gareev, S.P. Ivanova, L.A. Malov, and V.G. Soloviev, Nucl. Phys. A171, 134 (1971).

[5] A.V. Afanasjev, T.L. Khoo, S. Frauendorf, G.A. Lalazissis, I. Ahmad, to be published in Phys. Rev. C.

[6] V.V. Pashkevich, Nucl. Phys. A169, 275 (1971).

[7] F.A. Ivanyuk, H. Hofmann, V.V. Pashkevich, S. Yamaji, Phys. Rev. C55, 1730 (1997).

[8] F. Garcia, E. Garrote, M.-L. Yoneama, J.D.T. Arruda-Neto, J. Mesa, F. Bringas, J.F. Dias, V.P. Likhachev, O. Rodriguez and F. Guzmán, Eur. Phys. J. A6, 49 (1999).

[9] T.H.R. Skyrme, Phil. Mag. 1, 1043 (1956); D. Vauterin and D.M. Brink, Phys. Rev. C5, 626 (1972).

[10] J. Bartel, P. Quentin, M. Brack, C. Guet, and H.-B. Håakansson, Nucl. Phys. A386, 79 (1982).

[11] E.N. Shurshikov, Nucl. Data Sheets, 53, 601 (1988).

[12] V.G. Soloviev, Theory of Complex Nuclei (Pergamon Press, 1976).

[13] D. Van Neck, M. Waroquier, V. Van der Sluys, and K. Heide, Nucl. Phys. A563, 1 (1993). 
[14] C. Mahaux, P.F. Bortignon, R.A. Broglia, and C.H. Dasso, Phys. Rep. 120, 1 (1985). 


\section{FIGURE CAPTIONS}

Figure 1: MD for selected hole proton states in ${ }^{238} \mathrm{U}: \mathrm{SkM}^{*}$ (solid curve) and WS (dashed curve) results at equilibrium deformations as well as $\mathrm{SkM}^{*}$ results in the spherical limit (dotted curve).

Figure 2: MD for $K_{i}^{\pi}=1 / 2^{+}$hole proton states in ${ }^{238} \mathrm{U}$ : SkM* (solid curve) and WS (dashed curve) results.

Figure 3: MD at $k=0$ for the $K_{i}^{\pi}=1 / 2^{+}$hole proton states (the same as in figure 2). 


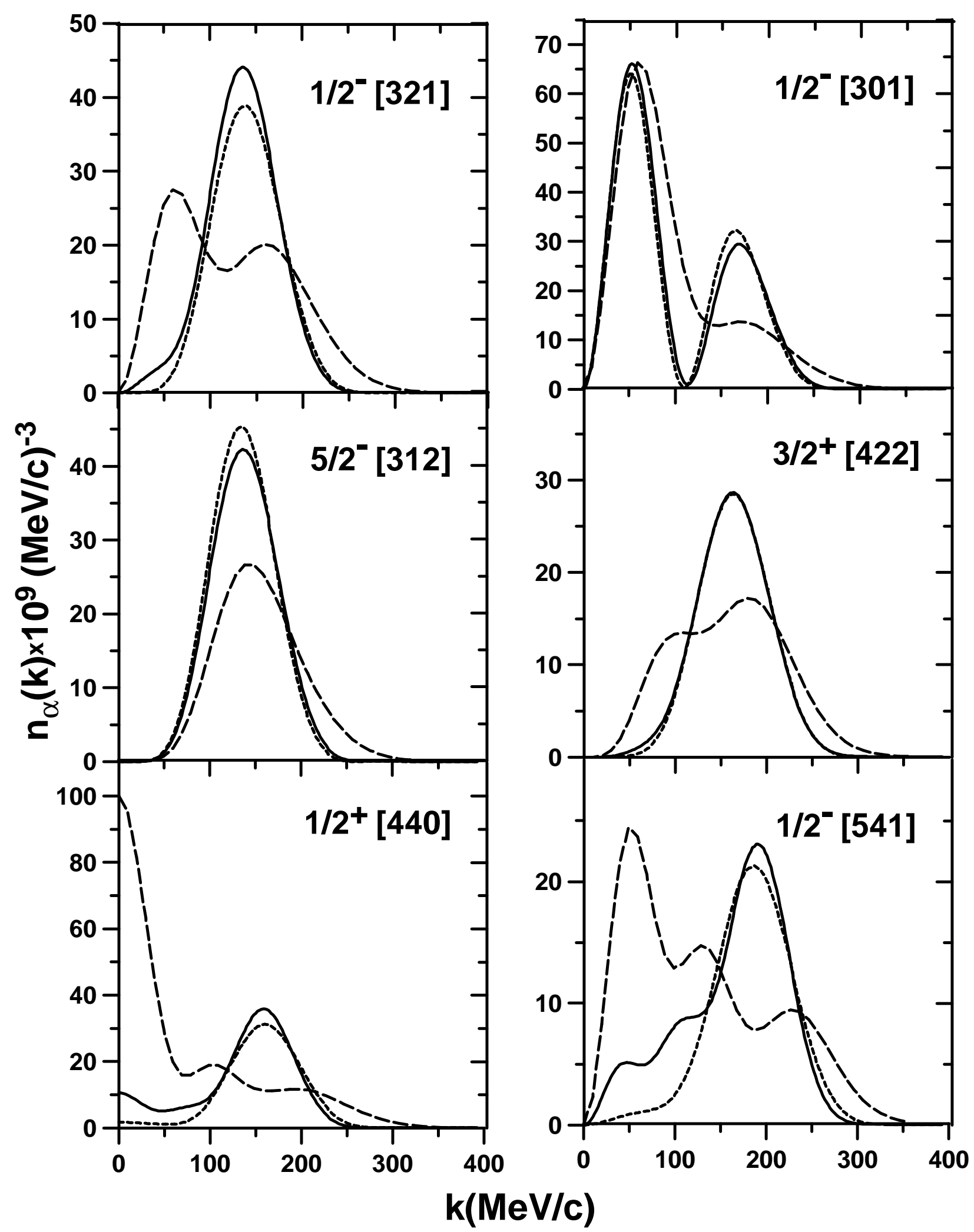




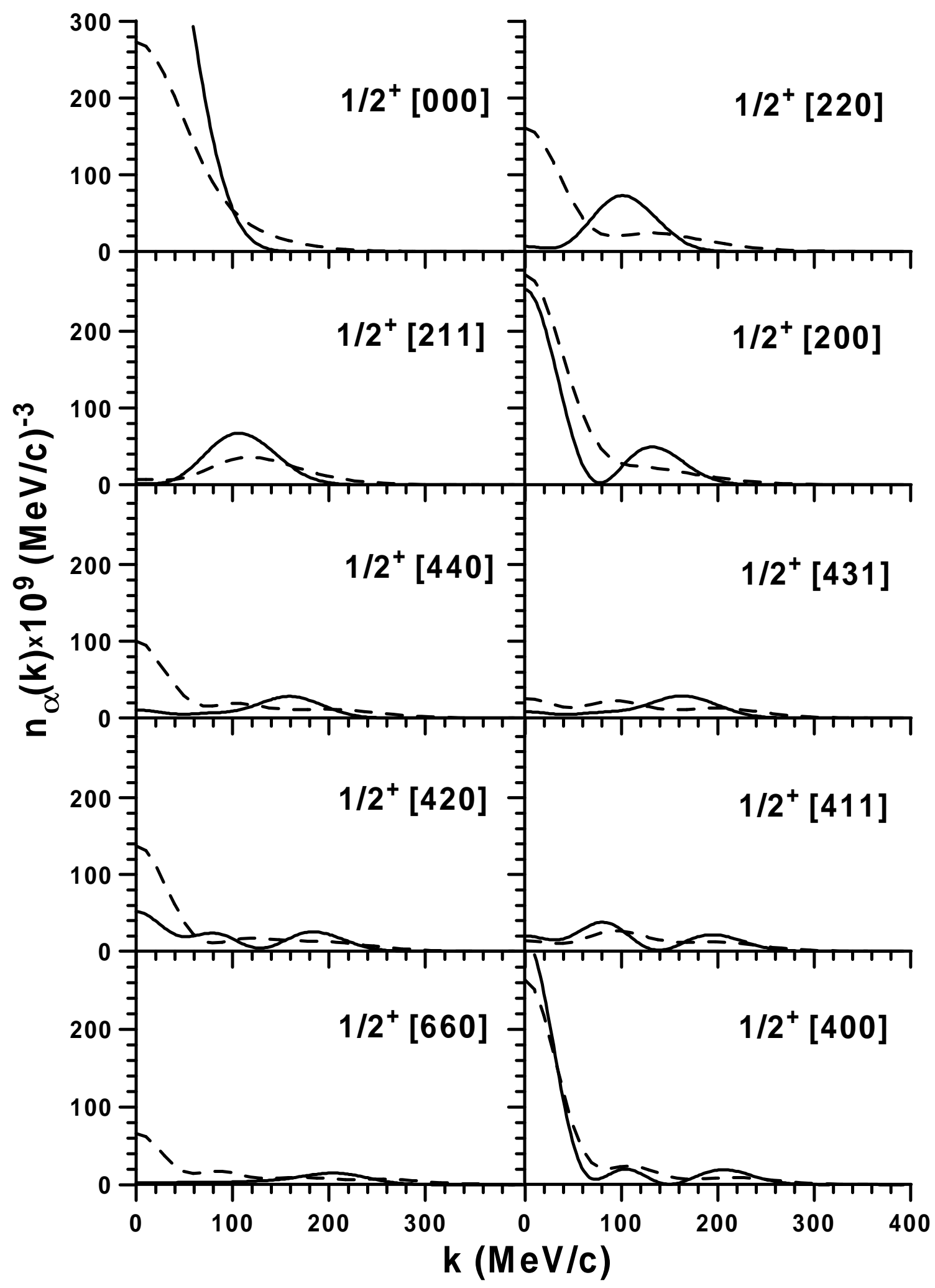




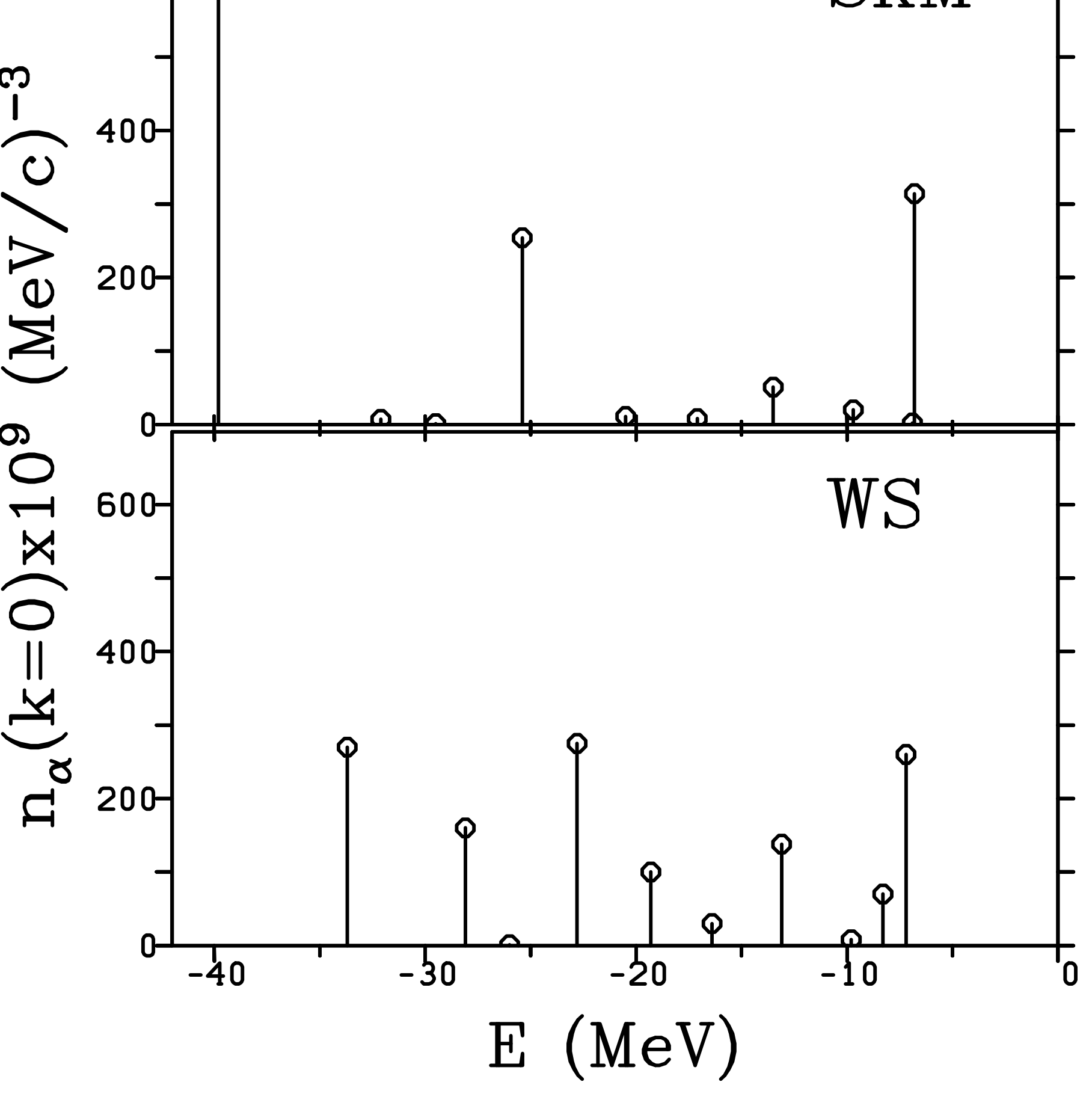

